\title{
IS COMPLETE SYSTEMATIC NODAL DISSECTION BY THORACOSCOPIC SURGERY POSSIBLE? A PROSPECTIVE TRIAL OF VIDEO-ASSISTED LOBECTOMY FOR CANCER OF THE RIGHT LUNG
}

Takashi Kondo, MD, Motoyasu Sagawa, MD, Tatsuo Tanita, MD, Masami Sato, MD, Sadafumi Ono, MD, Yuji Matsumura, MD, and Shigefumi Fujimura, MD, Sendai, Japan

Recently, video-assisted thoracic surgery (VATS) has been widely applied to various intrathoracic benign diseases. The procedure is apparently less invasive than any other conventional standard thoracotomy and is also cosmetically pleasing to patients. If VATS can be applied to the treatment of primary lung cancer, numerous benefits for patients can be expected, even if a small thoracotomy is needed to complete the pulmonary resection. However, one problem remains in the application of the procedure for surgical treatment of primary lung cancer, that is, the possibility of incomplete systematic nodal dissection. To clarify the feasibility of sufficient dissection with VATS, we conducted a prospective clinical trial in 6 patients with clinical stage I cancer of the right lung.

Patients and methods. Patients with stage I cancer of the right lung were chosen for the present assessment because the upper mediastinal and pretracheal nodes can be dissected without the difficulty that is encountered in cancer of the left lung. Inasmuch as this is the first trial, cases in which bronchoplasty or angioplasty was anticipated were excluded. The present study was approved by our institutional review board and complete informed consent on this trial of thoracoscopy for surgical treatment of lung cancer was obtained from each patient.

With a small skin incision 7 to $8 \mathrm{~cm}$ in length, the right fifth intercostal space was entered just below the lower angle of the scapular bone. Respiratory muscles and ribs were not severed. Two additional thoracoports were placed in the fourth and seventh intercostal spaces at the anterior and mid to posterior axillary lines, respectively. Through the small wound

From the Department of Thoracic Surgery, Institute of Development, Aging and Cancer, Tohoku University, Sendai, Japan.

Received for publication June 4, 1998; accepted for publication June 9, 1998.

Address for reprints: Takashi Kondo, MD, Department of Thoracic Surgery, Institute of Development, Aging and Cancer, Tohoku University, 4-1 Seiryomachi Aobaku, Sendai 980-8575, Japan.

J Thorac Cardiovasc Surg 1998;116:651-2

Copyright (C) 1998 by Mosby, Inc.

0022-5223/98 $\$ 5.00+0 \quad \mathbf{1 2 / 5 4 / 9 2 4 7 0}$
Table I. Profile of patients and the disease state in the present study

\begin{tabular}{lcccc}
\hline & $\begin{array}{c}\text { Primary } \\
\text { site }\end{array}$ & Pathology & cTNM & $\begin{array}{c}\text { Operative } \\
\text { procedure }\end{array}$ \\
\hline $1(71, \mathrm{M})$ & Upper lobe & Adenocarcinoma & T1 N0 M0 & RUML \\
$2(68, \mathrm{~F})$ & Upper lobe & Adenocarcinoma & T1 N0 M0 & RUL \\
$3(60, \mathrm{~F})$ & Upper lobe & Adenocarcinoma & T1 N0 M0 & RUML \\
4 (68, F) & Upper lobe & Adenocarcinoma & T1 N0 M0 & RUL \\
$5(79$, F) & Middle lobe & Adenocarcinoma & T1 N0 M0 & RML \\
$6(76$, F) & Upper lobe & Adenocarcinoma & T1 N0 M0 & RUL \\
\hline
\end{tabular}

$R U M L$, Right upper and middle lobectomy; RUL, right upper lobectomy; $R M L$, right middle lobectomy.

and the 2 thoracoports, a designated pulmonary lobe(s) was resected with hilar and mediastinal lymph node dissection (VATS lobectomy with lymph node dissection). In most cases, the pulmonary vein and bronchus were transected with staplers and branches of pulmonary artery were ligated and transected through the small thoracotomy wound. After VATS lobectomy with lymph node dissection, a standard thoracotomy was subsequently carried out by a different surgeon to complete systematic nodal dissection.

At VATS lobectomy, the number of dissected lymph nodes at each station and the weight of dissected tissues including fat tissue were recorded. The location and the number of remaining lymph nodes and the weight of dissected tissues found at additional thoracotomy were recorded as well. Percent of remaining nodes was calculated both for number and for weight. Operating time required for VATS lobectomy with lymph node dissection and the amount of bleeding during the procedure were also recorded.

Results. To date, 6 patients have been treated. The primary lesion was located in the upper lobe in 5 patients and in the middle lobe in the other patient. Upper lobectomy was performed in 4 patients and upper and middle lobectomy in 2 patients. Clinical stage was T1 N0 M0 in all patients (Table I). 
Table II. Time needed for VATS procedure and amount of bleeding during VATS; number of lymph nodes resected by VATS and number and location of residual lymph nodes found at subsequent standard thoracotomy; weight of dissected and residual tissues, including lymph nodes, resected by VATS

\begin{tabular}{|c|c|c|c|c|c|c|}
\hline & Patient 1 & Patient 2 & Patient 3 & Patient 4 & Patient 5 & Patient 6 \\
\hline Operating time (min) & 260 & 190 & 250 & 140 & 200 & 240 \\
\hline Blood loss (g) & 140 & 70 & 165 & 100 & 50 & 65 \\
\hline \multicolumn{7}{|l|}{ No. of lymph nodes } \\
\hline VATS dissection & 43 & 27 & 28 & 37 & 48 & 46 \\
\hline Subsequent standard dissection & 6 & 0 & 0 & 0 & 0 & 2 \\
\hline Remaining lymph nodes & No. 11, No. $3^{*}$ & - & - & - & - & No. 9 \\
\hline \multicolumn{7}{|l|}{$\begin{array}{l}\text { Weight of dissected and residual } \\
\text { tissues }\end{array}$} \\
\hline VATS (g) & 16.1 & 5.4 & 8.9 & 5.0 & 8.0 & 9.8 \\
\hline Subsequent standard dissection & 0.6 & 0 & 0 & 0 & 0 & 0.2 \\
\hline Remaining lymph nodes (\%) & 3.5 & 0 & 0 & 0 & 0 & 2.0 \\
\hline
\end{tabular}

*Retrotracheal node.

The time required for VATS lobectomy with lymph node dissection was 140 to 260 minutes and the amount of bleeding was 50 to $165 \mathrm{~g}$ (Table II). The average operating time and the amount of bleeding were 213 minutes and $98 \mathrm{~g}$, respectively. The postoperative course was uneventful in all patients.

The number of dissected lymph nodes by VATS lobectomy was 27 to 48, and remaining lymph nodes were found in 2 patients at additional thoracotomy. The weight of dissected tissue by VATS lobectomy was 5.0 to $16.1 \mathrm{~g}$. In the 2 patients in whom remaining lymph nodes were found at subsequent standard thoracotomy, the weight of the additionally dissected tissue was 0.6 and $0.2 \mathrm{~g}$ and the percentage of lymph nodes remaining was $3.5 \%$ and $2 \%$, respectively (Table II). Overall percentage of nodes remaining after systematic nodal dissection by VATS lobectomy was $3.4 \%$ according to the number and $1.5 \%$ according to the weight.

Discussion. Recent advances and improvements in devices and equipment for endoscopic surgery enabled us to extend its application to various disorders, not only benign conditions but malignant diseases as well. Recently, several reports have described the application of VATS to the early stages of primary lung cancer. ${ }^{1,2}$ Although standard and VATS procedures have yielded equivalent survivals, ${ }^{3}$ the absolute indications for VATS for patients with lung cancer have yet to be defined, because no scientific studies on the validity of the procedure for systematic nodal dissection in primary lung cancer have been reported. In this study, residual lymph nodes were found after the VATS procedure in 2 of 6 patients; however, the number and the weight of those nodes were unexpectedly small. Operative time and the amount of bleeding with the VATS procedure were not unreasonable when compared with the standard procedure.

Several techniques for VATS lobectomy have been presented to date. ${ }^{4,5}$ The axillary approach has been commonly used in these previous reports. Although this approach is useful in dissecting hilar structures, it does not seem to be suitable for mediastinal lymph node dissection, especially for dissecting subcarinal nodes. In this study, we have chosen the posterior approach for the small thoracotomy. From our experiences, the subcarinal, pretracheal, and highest mediastinal lymph nodes were easily dissected through this approach. In addition, no special devices for thoracoscopic surgery were necessary to perform nodal dissection because of the short distance between the small thoracotomy incision and the mediastinum and the direct visibility of the mediastinum through the incision. Complete en bloc systematic nodal dissection was possible with ordinary surgical devices using the present procedure in a right-sided operation.

Thus we conclude that the standard lobectomy with systematic nodal dissection for cancer of the right lung is technically feasible and can be performed safely through a small thoracotomy with the aid of thoracoscope. The present procedure can be applied to cancer of the right lung that meets the indications for standard surgical techniques.

\section{REFERENCES}

1. Landreneau RJ, Mack MJ, Dowling RD, Luketich JD, Keenan RJ, Ferson PF, et al. The role of thoracoscopy in lung cancer management. Chest 1998;113:6S-12S.

2. Yim AP, Liu HP. Thoracoscopic major lung resection: indications, technique, and early results-experience from two centers in Asia. Surg Laparosc Endosc 1997;7:241-4.

3. Iwasaki A, Shirakusa T, Kawahara K, Yoshinaga Y, Okabayashi $\mathrm{K}$, Shiraishi T. Is video-assisted thoracoscopic surgery suitable for resection of primary lung cancer? Thorac Cardiovasc Surg 1997;45:13-5.

4. Iwasaki M, Nishiumi N, Maitani F, Kaga K, Ogawa J, Inoue H. Thoracoscopic surgery for lung cancer using the two small skin incisional method: two windows method. J Cardiovasc Surg 1996;37:79-81.

5. McKenna R Jr. VATS lobectomy with mediastinal lymph node sampling or dissection. Chest Surg Clin North Am 1995;5:22332. 